\title{
SOME HOMOGENEOUS BIANCHI TYPE IX VISCOUS FLUID COSMOLOGICAL MODELS WITH A VARYING $\Lambda$
}

\author{
ANIRUDH PRADHAN* and SUDHIR KUMAR SRIVASTAV \\ Department of Mathematics, Hindu Post-graduate College, Zamania-232 331, \\ Ghazipur, U. P., India; \\ E-mail:acpradhan@yahoo.com,pradhan@iucaa.ernet.in
}

\author{
MAHESH KUMAR YADAV \\ Department of Mathematics, Stani Memorial College (IIRM Campus), \\ Mansarovar, Jaipur-302 020, India
}

September 17, 2018

\begin{abstract}
Some Bianchi type IX viscous fluid cosmological models are investigated. To get a solution a supplementary condition between metric potentials is used. The viscosity coefficient of bulk viscous fluid is assumed to be a power function of mass density whereas the coefficient of shear viscosity is considered as proportional to scale of expansion in the model. The cosmological constant $\Lambda$ is found to be positive and is a decreasing function of time which is supported by results from recent supernova observations. Some physical and geometric properties of the models are also discussed.
\end{abstract}

Keywords: Cosmology; Bianchi type IX universe; Viscous fluid models; variable cosmological constant.

\section{Introduction}

Bianchi type IX cosmological models are important and interesting in the sense that these models allow not only expansion but also rotation and shear and in general are anisotropic. Bianchi type IX universes include closed FRW models. The homogeneous and isotropic FRW cosmological models which are used to describe standard cosmological models, are particular case of Bianchi type I, V, and IX space-times according as the constant curvature of the physical three-space, $t=$ constant, is zero, negative or positive. In these models, neutrino viscosity explains the large radiation entropy in the universe and the degree of isotropy of the cosmic background radiation. The standard cosmological models are too restrictive because of the insistence on the isotropy of the physical three-space and several attempts have been made to study non-standard cosmological models e. g. MacCallum (1979); Narlikar

* Corresponding Author

(C) 2018 Kluwer Academic Publishers. Printed in the Netherlands. 
and Kembhavi (1980); and Narlikar 1983). It is therefore interesting to carry out the detailed studies of gravitational fields which are described by space-time of various Bianchi types. Vaidya and Patel (1986) have studied spatially homogeneous space-time of Bianchi type IX and they have outlined general scheme for the derivation of exact solutions of Einstein's field equations in presence of perfect fluid and pure radiation fields. Krori te al. (1990); Chakraborty and Nandy (1992) have investigated cosmological models of Bianchi type II, VIII and IX. There are many other researchers (Uggla and Zur-Muhlem, 1990; Burd, Buric Ellis, 1990; King, 1991; Paternoga and Graham, 1996), who have studied Bianchi type IX space-time in different context. Recently Bali and Dave (2001, 2003) have investigated Bianchi type IX string cosmological models.

Models with a dynamic cosmological term $\Lambda(t)$ are becoming popular as they solve the cosmological constant problem in a natural way. There is significant observational evidence for the detection of Einstein's cosmological constant, $\Lambda$ or a component of material content of the universe that varies slowly with time and space and so acts like $\Lambda$. Recent cosmological observations by High -z Supernova Team and Supernova Cosmological Project (Garnavich et al., 1998; Perlmutter et al., 1997, 1998, 1999; Riess et al., 1998; Schmidt et al., 1998) suggest the existence of a positive cosmological constant $\Lambda$ with magnitude $\Lambda\left(G \hbar / c^{3}\right) \approx 10^{-123}$. These observations on magnitude and red-shift of type Ia supernova suggest that our universe may be a accelerating with a large function of the cosmological density in the form of the cosmological $\Lambda$-term. Earlier researchers on this topic, are contained in Zeldovich (1968), Weinberg (1972), Dolgov (1983, 1990), Bertolami (1986), Ratra and Peebles (1988), Carroll, Press and Turner (1992). Some of the recent discussions on the cosmological constant "problem" and consequence on cosmology with a time-varying cosmological constant have been discussed by Dolgov $(1993,1997)$, Tsagas and Maartens (2000), Sahni and Starobinsky (2000), Peebles (2002), Padmanabhan (2003), Vishwakarma (1999, 2000, 2001, 2002), and Pradhan et al. (2001, 2002, 2003). This motivates us to study the cosmological models in which $\Lambda$ varies with time.

The majority of the studies in cosmology involve a perfect fluid. However, observed physical phenomena such as the large entropy per baryon and the remarkable degree of isotropy of the cosmic microwave background radiation suggests analysis of dissipative effects in cosmology. Furthermore, there are several processes which are expected to give rise to viscous effects. These are the decoupling of neutrinos during the 
radiation era and the decoupling of radiation and matter during the recombination era. Bulk viscosity is associated with the GUT phase transition and string creation. The model studied by Murphy (1973) possessed an interesting feature in that the big bang type of singularity of infinite space-time curvature does not occur to be a finite past. However, the relationship assumed by Murphy between the viscosity coefficient and the matter density is not acceptable at large density. The effect of bulk viscosity on the cosmological evolution has been investigated by a number of authors in the framework of general theory of relativity (Padmanabhan and Chitre, 1987; Johri and Sudarshan, 1988; Maartens, 1995; Zimdahl, 1996; Pradhan, Sarayakar and Beesham, 1997; Kalyani and Singh (1997; Singh, Beesham and Mbokazi, 1998; Pradhan et al., 2001, 2002). This motivates to study cosmological bulk viscous fluid model.

Recently Bali and Yadav (2002) has investigated Bianchi type IX viscous fluid cosmological models. Motivated by the situations discussed above, in this paper, we shall focus upon the problem with varying cosmological constant in presence of bulk and shear viscous fluid in an expanding universe. We do this by extending the work of Bali and Yadav (2002) by including varying cosmological constant and the coefficient of bulk viscosity as function of time. This paper is organized as follows. The metric and the field equations are presented in section 2. In section 3 , we deal with the solution of the field equations in presence of viscous fluid. Section 4 includes the solution of some particular models whereas in section 5 , we deal with some special models. In section 6 , we have given the concluding remarks.

\section{The metric and field equations}

We consider the Bianchi type IX metric in the form

$$
\begin{gathered}
d s^{2}=-d t^{2}+A^{2} d x^{2}+B^{2} d y^{2}+\left(B^{2} \sin ^{2} y+A^{2} \cos ^{2} y\right) d z^{2} \\
-2 A^{2} \cos y d x d z
\end{gathered}
$$

where $\mathrm{A}$ and $\mathrm{B}$ are functions of $t$ only.

The Einstein's field equations (in gravitational units $c=1, G=1$ ) read as

$$
R_{i}^{j}-\frac{1}{2} R g_{i}^{j}+\Lambda g_{i}^{j}=-8 \pi T_{i}^{j}
$$

where $R_{i}^{j}$ is the Ricci tensor; $R=g^{i j} R_{i j}$ is the Ricci scalar; and $T_{i}^{j}$ is the stress energy-tensor in the presence of bulk stress given by Landau 
and Lifshitz (1963)

$$
\begin{aligned}
T_{i}^{j}=(\rho+p) v_{i} v^{j} & +p g_{i}^{j}-\eta\left(v_{i ;}^{j}+v_{; i}^{j}+v^{j} v^{l} v_{i ; l}+v_{i} v^{l} v_{; l}^{j}\right) \\
& -\left(\xi-\frac{2}{3} \eta\right) \theta\left(g_{i}^{j}+v_{i} v^{j}\right) .
\end{aligned}
$$

Here $\rho, p, \eta$ and $\xi$ are the energy density, isotropic pressure, coefficient of shear viscosity and bulk viscous coefficient respectively and $v_{i}$ is the flow vector satisfying the relations

$$
g_{i j} v^{i} v^{j}=-1 .
$$

The semicolon $(;)$ indicates covariant differentiation. We choose the coordinates to be comoving, so that

$$
v^{1}=0=v^{2}=v^{3}, v^{4}=1
$$

The Einstein's field equations (2) for the line element (1) has been set up as

$$
\begin{gathered}
-8 \pi\left[p-2 \eta \frac{A_{4}}{A}-\left(\xi-\frac{2}{3} \eta\right) \theta\right]=\frac{2 B_{44}}{B}+\frac{B_{4}^{2}}{B^{2}}+\frac{1}{B^{2}}-\frac{3 A^{2}}{4 B^{4}}+\Lambda \\
-8 \pi\left[p-2 \eta \frac{B_{4}}{B}-\left(\xi-\frac{2}{3} \eta\right) \theta\right]=\frac{A_{44}}{A}+\frac{A_{4} B_{4}}{A B}+\frac{B_{44}}{B}+\frac{A^{2}}{4 B^{4}}+\Lambda, \\
8 \pi \rho=\frac{2 A_{4} B_{4}}{A B}+\frac{B_{4}^{2}}{B^{2}}+\frac{1}{B^{2}}-\frac{A^{2}}{4 B^{4}}+\Lambda
\end{gathered}
$$

where the suffix 4 at the symbols $A$ and $B$ denotes ordinary differentiation with respect to $t$ and $\theta$ is the scalar of expansion given by

$$
\theta=v_{; i}^{i}
$$

\section{Solution of the field equations}

Equations (6) - (8) are three independent equations in seven unknowns $A, B, \rho, p, \eta, \xi$ and $\Lambda$. For the complete determinacy of the system, we need four extra conditions.

Firstly we assume a relation in metric potential as

$$
A=B^{m}
$$

and secondly we assume that the coefficient of shear viscosity is proportional to the scale of expansion, i. e.,

$$
\eta \propto \theta
$$


where $m$ is a real number.

Eqs. (6) and (7) lead to

$$
\frac{B_{44}}{B}+\frac{B_{4}^{2}}{B^{2}}-\frac{A_{44}}{A}-\frac{A_{4} B_{4}}{A B}-\frac{A^{2}}{B^{4}}+\frac{1}{B^{2}}=16 \pi \eta\left(\frac{A_{4}}{A}-\frac{B_{4}}{B}\right) .
$$

Condition (11) leads to

$$
\eta=\ell\left(\frac{A_{4}}{A}+\frac{2 B_{4}}{B}\right)
$$

where $\ell$ is a proportionality constant.

Equations (12) together with (10) and (13) leads to

$$
B B_{44}+\alpha B_{4}{ }^{2}=\frac{B^{2(m-1)}}{(1-m)}-\frac{1}{(1-m)}, \quad m \neq 1,
$$

which can be rewritten as

$$
\frac{d}{d B}\left(f^{2}\right)+\frac{2 \alpha}{B}\left(f^{2}\right)=\frac{2 B^{2 m-3}}{(1-m)}-\frac{2}{(1-m) B}
$$

where

$$
\alpha=(1+m)-16 \pi \ell \frac{\left(m^{2}+m-2\right)}{(1-m)}
$$

and

$$
B_{4}=f(B)
$$

From (15), we obtain

$$
\left(\frac{d B}{d t}\right)^{2}=\left[\frac{B^{2(m-1)}}{(1-m)(m+\alpha-1)}+\frac{\beta}{B^{2 \alpha}}-\frac{1}{\alpha(1-m)}\right],
$$

where $\beta$ is a constant of integration. After a suitable transformation of coordinates, the metric (1) reduces to the form

$$
\begin{gathered}
d s^{2}=-\left[\frac{T^{2(m-1)}}{(1-m)(m+\alpha-1)}+\frac{\beta}{T^{2 \alpha}}-\frac{1}{\alpha(1-m)}\right]^{-1} d T^{2}+T^{2 m} d x^{2}+ \\
T^{2} d y^{2}+\left(T^{2} \sin ^{2} y+T^{2 m} \cos ^{2} y\right) d z^{2}-2 T^{2 m} \cos y d x d y
\end{gathered}
$$

where $B=T$.

The pressure and density for the model (19) are given by

$$
8 \pi p=K_{1} T^{2(m-2)}+\frac{K_{2}}{T^{2}}+\frac{\beta K_{3}}{T^{2(\alpha+1)}}+
$$




$$
\begin{gathered}
8 \pi \xi(m+2) \sqrt{\left[\frac{T^{2(m-2)}}{(m+\alpha-1)(1-m)}+\frac{\beta}{T^{2(\alpha+1)}}-\frac{1}{\alpha(1-m) T^{2}}\right]}-\Lambda \\
8 \pi \rho=K_{4} T^{2(m-2)}+\frac{\beta(2 m+1)}{T^{2(\alpha+1)}}+\frac{K_{5}}{T^{2}}+\Lambda
\end{gathered}
$$

where

$K_{1}=\frac{\left(-m^{2}\right)(64 \pi \ell+21)-m(64 \pi \ell-3 \alpha+6)+(128 \pi \ell-3 \alpha+15)}{12(m+\alpha-1)(1-m)}$,

$K_{2}=\frac{m^{2}(16 \pi \ell+3)+m(16 \pi \ell)-32 \pi \ell}{3 \alpha(1-m)}$,

$K_{3}=\frac{1}{3}\left[\left(-m^{2}\right)(16 \pi \ell+3)-m(16 \pi \ell-3 \alpha)+(32 \pi \ell+3 \alpha)\right]$,

$K_{4}=\frac{m^{2}+m(\alpha+6)-(\alpha-5)}{4(m+\alpha-1)(1-m)}$,

$K_{5}=\frac{(-m)(\alpha+2)+(\alpha-1)}{\alpha(1-m)}$.

For the specification of $\xi$, we assume that the fluid obeys an equation of state of the form

$$
p=\gamma \rho
$$

where $\gamma(0 \leq \gamma \leq 1)$ is constant.

Thus, given $\xi(t)$ we can solve for the cosmological parameters. In most of the investigation involving bulk viscosity is assumed to be a simple power function of the energy density (Pavon, 1991; Maartens, 1995; Zimdahl, 1996)

$$
\xi(t)=\xi_{0} \rho^{n},
$$

where $\xi_{0}$ and $n$ are constants. If $n=1$, Equation (23) may correspond to a radiative fluid (Weinberg, 1972). However, more realistic models (Santos, 1985) are based on $n$ lying in the regime $0 \leq n \leq \frac{1}{2}$.

On using (23) in (20), we obtain

$$
\begin{gathered}
8 \pi p=K_{1} T^{2(m-2)}+\frac{K_{2}}{T^{2}}+\frac{\beta K_{3}}{T^{2(\alpha+1)}}+ \\
8 \pi \xi_{0} \rho^{n}(m+2) \sqrt{\left[\frac{T^{2(m-2)}}{(m+\alpha-1)(1-m)}+\frac{\beta}{T^{2(\alpha+1)}}-\frac{1}{\alpha(1-m) T^{2}}\right]}-\Lambda .
\end{gathered}
$$

\subsection{Model I: Solution for $\xi=\xi_{0}$}

When $n=0$, Equation (23) reduces to $\xi=\xi_{0}=$ constant. Hence in this case Equation (24), with the use of (21) and (22), leads to

$$
8 \pi(1+\gamma) \rho=\left(K_{1}+K_{4}\right) T^{2(m-2)}+\frac{\left(K_{2}+K_{5}\right)}{T^{2}}+\frac{\beta\left(K_{3}+2 m+1\right)}{T^{2(\alpha+1)}}
$$




$$
+8 \pi \xi_{0}(m+2) \sqrt{\left[\frac{T^{2(m-2)}}{(m+\alpha-1)(1-m)}+\frac{\beta}{T^{2(\alpha+1)}}-\frac{1}{\alpha(1-m) T^{2}}\right]} .
$$

Eliminating $\rho(t)$ between Equations (21) and (25), we have

$$
\begin{aligned}
& (1+\gamma) \Lambda=\left(K_{1}-\gamma K_{4}\right) T^{2(m-2)}+\frac{\left(K_{2}-\gamma K_{5}\right)}{T^{2}}+\frac{\beta\left(K_{3}-\gamma(2 m+1)\right)}{T^{2(\alpha+1)}} \\
& \quad+8 \pi \xi_{0}(m+2) \sqrt{\left[\frac{T^{2(m-2)}}{(m+\alpha-1)(1-m)}+\frac{\beta}{T^{2(\alpha+1)}}-\frac{1}{\alpha(1-m) T^{2}}\right]} .
\end{aligned}
$$

\subsection{Model II: SOlution FOR $\xi=\xi_{0} \rho$}

When $n=1$, Equation (23) reduces to $\xi=\xi_{0} \rho$. Hence in this case Equation (24), with the use of (21) and (22), leads to

$$
\begin{gathered}
8 \pi \rho=\frac{1}{\left[1+\gamma-\xi_{0}(m+2) \sqrt{\left[\frac{T^{2(m-2)}}{(m+\alpha-1)(1-m)}+\frac{\beta}{\left.T^{2(\alpha+1)}-\frac{1}{\alpha(1-m) T^{2}}\right]}\right]}\right.} \times \\
{\left[K_{1} T^{2(m-2)}+\frac{K_{2}}{T^{2}}+\frac{\beta K_{3}}{\left.T^{2(\alpha+1)}\right] .}\right.}
\end{gathered}
$$

Eliminating $\rho(t)$ between Equations (21) and (27), we have

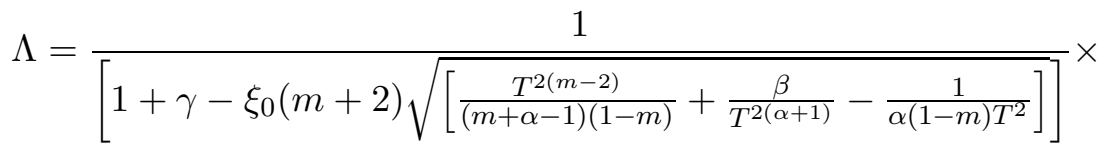

$$
\begin{aligned}
& {\left[K_{1} T^{2(m-2)}+\frac{K_{2}}{T^{2}}+\frac{\beta K_{3}}{T^{2(\alpha+1)}}\right]-\left(K_{4} T^{2(m-2)}+\frac{K_{5}}{T^{2}}+\frac{\beta(2 m+1)}{T^{2(\alpha+1)}}\right) .}
\end{aligned}
$$

From Equations (26) and (28), we observe that when $\alpha>0$ and $m<2$, the positive cosmological constant is a decreasing function of time and approaches a small value in the present epoch.

Some Physical Aspects of the Models:

With regard to the kinematical properties of the velocity vector $v^{i}$ in the metric (19), a straight forward calculation leads to the following expressions for the scalar of expansion $(\theta)$ and for the shear $(\sigma)$ of the fluid.

$$
\theta=(m+2)\left[\sqrt{\left[\frac{T^{2(m-2)}}{(m+\alpha-1)(1-m)}+\frac{\beta}{T^{2(\alpha+1)}}-\frac{1}{\alpha(1-m) T^{2}}\right]}\right]
$$




$$
\sigma=\sqrt{\frac{2}{3}}(1-m)\left[\sqrt{\left[\frac{T^{2(m-2)}}{(m+\alpha-1)(1-m)}+\frac{\beta}{T^{2(\alpha+1)}}-\frac{1}{\alpha(1-m) T^{2}}\right]}\right]
$$

For $\alpha>0$ and $m<2$, the expansion factor $\theta$ is a decreasing function of $T$ and approaches, asymptotically to zero with $\rho$ and $p$ also approaching to zero as $T \rightarrow \infty$.

\section{Particular Models}

If we set $m=2$, then the geometry of the space-time (19) reduces to the form

$$
\begin{aligned}
& -\left[\frac{\beta}{T^{2(3+64 \pi \ell)}}-\frac{T^{2}}{4(1+16 \pi \ell)}+\frac{1}{(3+64 \pi \ell)}\right] d T^{2} \\
& +T^{4} d x^{2}+T^{2} d y^{2}+\left(T^{2} \sin ^{2} y+T^{4} \cos ^{2} y\right) d z^{2}-2 T^{4} \cos y d x d z,
\end{aligned}
$$

where $\beta$ is an integrating constant.

The pressure and density of the model (31) are given by

$$
\begin{gathered}
8 \pi p=\frac{\beta(15+512 \pi \ell)}{3 T^{8(1+16 \pi \ell)}-\frac{4(3+16 \pi \ell)}{3(3+64 \pi \ell) T^{2}}+\frac{(9+8 \pi \ell)}{6(1+16 \pi \ell)}} \\
+(32 \pi \xi) \sqrt{\left[\frac{\beta}{T^{8(1+16 \pi \ell)}}+\frac{1}{(3+64 \pi \ell) T^{2}}-\frac{1}{4(1+16 \pi \ell)}\right]}-\Lambda \\
8 \pi \rho=\frac{5 \beta}{T^{8(1+16 \pi \ell)}+\frac{8(1+8 \pi \ell)}{(3+64 \pi \ell) T^{2}}-\frac{(3+8 \pi \ell)}{2(1+16 \pi \ell)}+\Lambda .}
\end{gathered}
$$

\subsection{Model I: Solution For $\xi=\xi_{0}$}

When $n=0$, Equation (23) reduces to $\xi=\xi_{0}=$ constant. Hence in this case Equation (32), with the use of (33) and (22), leads to

$$
\begin{aligned}
& 8 \pi(1+\gamma) \rho=\frac{2 \beta(15+256 \pi \ell)}{3 T^{8(1+16 \pi \ell)}+\frac{4(15+32 \pi \ell)}{3(3+64 \pi \ell) T^{2}}-\frac{16 \pi \ell}{3(1+16 \pi \ell)}} \\
& +\left(32 \pi \xi_{0}\right) \sqrt{\left[\frac{\beta}{T^{8(1+16 \pi \ell)}}+\frac{1}{(3+64 \pi \ell) T^{2}}-\frac{1}{4(1+16 \pi \ell)}\right]}
\end{aligned}
$$

Eliminating $\rho(t)$ between (33) and (34), we obtain

$$
(1+\gamma) \Lambda=\frac{\beta(15+512 \pi \ell-15 \gamma)}{3 T^{8(1+16 \pi \ell)}}-\frac{4(3+16 \pi \ell+6(3+8 \pi \ell) \gamma)}{3(3+64 \pi \ell) T^{2}}+\frac{(9-8 \pi \ell+3(3+8 \pi \ell) \gamma)}{6(1+16 \pi \ell)}
$$




$$
+\left(32 \pi \xi_{0}\right) \sqrt{\left[\frac{\beta}{\left.T^{8(1+16 \pi \ell)}+\frac{1}{(3+64 \pi \ell) T^{2}}-\frac{1}{4(1+16 \pi \ell)}\right]}\right.}
$$

\subsection{Model II: Solution for $\xi=\xi_{0} \rho$}

When $n=1$, Equation (23) reduces to $\xi=\xi_{0} \rho$. Hence in this case Equation (32), with the use of (33) and (22), leads to

$$
\begin{aligned}
\rho= & \frac{1}{8 \pi\left[1+\gamma-4 \xi_{0} \sqrt{\left[\frac{\beta}{T^{8(1+16 \pi \ell)}}+\frac{1}{(3+64 \pi \ell) T^{2}}-\frac{1}{4(1+16 \pi \ell)}\right]}\right]} \times \\
& \frac{2 \beta(15+256 \pi \ell)}{3 T^{8(1+16 \pi \ell)}}+\frac{4(15+32 \pi \ell)}{3(3+64 \pi \ell) T^{2}}-\frac{16 \pi \ell}{3(1+16 \pi \ell)}
\end{aligned}
$$

Eliminating $\rho(t)$ between (33) and (36), we obtain

$$
\begin{aligned}
\Lambda= & \frac{1}{\left[1+\gamma-4 \xi_{0} \sqrt{\left[\frac{\beta}{T^{8(1+16 \pi \ell)}}+\frac{1}{(3+64 \pi \ell) T^{2}}-\frac{1}{4(1+16 \pi \ell)}\right]}\right.} \times \\
& {\left[\frac{2 \beta(15+256 \pi \ell)}{\left.3 T^{8(1+16 \pi \ell)}+\frac{4(15+32 \pi \ell)}{3(3+64 \pi \ell) T^{2}}-\frac{16 \pi \ell}{3(1+16 \pi \ell)}\right]}\right.} \\
& -\frac{5 \beta}{T^{8(1+16 \pi \ell)}}-\frac{8(1+8 \pi \ell)}{(3+64 \pi \ell) T^{2}}+\frac{(3+8 \pi \ell)}{2(1+16 \pi \ell)}+\Lambda .
\end{aligned}
$$

From Equations (35) and (37), we observe that the positive cosmological constant is a decreasing function of time and approaches a small value in the present epoch.

\section{Some Physical Aspects of the Models:}

The expansion $(\theta)$ and the $\operatorname{shear}(\sigma)$ in the model (31) are given by

$$
\begin{aligned}
\theta & =4 \sqrt{\left[\frac{\beta}{\left.T^{8(1+16 \pi \ell)}+\frac{1}{(3+64 \pi \ell) T^{2}}-\frac{1}{4(1+16 \pi \ell)}\right]}\right.} \\
\sigma & =\sqrt{\frac{2}{3}\left[\frac{\beta}{\left.T^{8(1+16 \pi \ell)}+\frac{1}{(3+64 \pi \ell) T^{2}}-\frac{1}{4(1+16 \pi \ell)}\right]}\right.}
\end{aligned}
$$

The expansion factor $\theta$ in the model is a decreasing function of $T$. Since $\lim _{T \rightarrow \infty} \frac{\sigma}{\theta} \neq 0$, hence the models do not approach isotropy for large values of $T$. The model have singularity at $T=0$ which is real physical singularity. 


\section{Special Models}

If we set $m=2$ and $\ell=-\frac{1}{32 \pi}$, Equation (18) leads to

$$
\frac{\sqrt{2} B d B}{\sqrt{\left(2 B^{2}-B^{4}+2 \beta\right)}}=d t,
$$

which on integration gives

$$
B^{2}=1+M \sin (\sqrt{2} t+2 N),
$$

where $M=\sqrt{2 \beta+1}$ and $N$ ia a constant of integration. Hence, we obtain

$$
A=B^{2}=1+M \sin (\sqrt{2} t+2 N),
$$

Using the transformations

$$
\begin{gathered}
\sqrt{2} t+2 N=T, \\
x=X, \\
y=Y, \\
z=Z,
\end{gathered}
$$

the metric (1) takes the form

$$
\begin{gathered}
d s^{2}=-\frac{d T^{2}}{2}+(1+M \sin T)^{2} d X^{2}+(1+M \sin T) d Y^{2}+ \\
{\left[(1+M \sin T) \sin ^{2} Y+(1+M \sin T)^{2} \cos ^{2} Y\right] d Z^{2}} \\
-2(1+M \sin T)^{2} \cos Y d X d Z
\end{gathered}
$$

The pressure and density for the model (44) are given by

$$
\begin{gathered}
8 \pi p=\frac{16 \sqrt{2} \pi \xi M \cos T}{(1+M \sin T)}+\frac{\left[35 M^{2} \sin ^{2} T+30 M \sin T-2 M^{2}-3\right]}{12(1+M \sin T)^{2}}-\Lambda \\
8 \pi \rho=\frac{\left[3+10 M^{2}+2 M \sin T-11 M^{2} \sin ^{2} T\right]}{4(1+M \sin T)^{2}}+\Lambda
\end{gathered}
$$

\subsection{Model I: $\quad$ Solution for $\xi=\xi_{0}$}

When $n=0$, Equation (23) reduces to $\xi=\xi_{0}=$ constant. Hence in this case Equation (45), with the use of (46) and (22), leads to

$8 \pi(1+\gamma) \rho=\frac{16 \sqrt{2} \pi \xi_{0} M \cos T}{(1+M \sin T)}+\frac{\left[M^{2} \sin ^{2} T+18 M \sin T+14 M^{2}+3\right]}{6(1+M \sin T)^{2}}$. 
Eliminating $\rho(t)$ between (46) and (47), we obtain

$$
\begin{gathered}
(1+\gamma) \Lambda=\frac{16 \sqrt{2} \pi \xi_{0} M \cos T}{(1+M \sin T)}+\frac{\left[35 M^{2} \sin ^{2} T+30 M \sin T-2 M^{2}-3\right]}{12(1+M \sin T)^{2}} \\
-\frac{\left[3+10 M^{2}+2 M \sin T-11 M^{2} \sin ^{2} T\right] \gamma}{4(1+M \sin T)^{2}}
\end{gathered}
$$

\subsection{Model II: $\quad$ Solution FOR $\xi=\xi_{0} \rho$}

When $n=1$, Equation (23) reduces to $\xi=\xi_{0} \rho$. Hence in this case Equation (45), with the use of (46) and (22), leads to

$$
\begin{gathered}
8 \pi \rho=\frac{1}{\left[1+\gamma-\frac{2 \sqrt{2} \xi_{0} M \cos T}{(1+M \sin T)}\right]} \times \\
\frac{\left[M^{2} \sin ^{2} T+18 M \sin T+14 M^{2}+3\right]}{6(1+M \sin T)^{2}} .
\end{gathered}
$$

Eliminating $\rho(t)$ between (46) and (49), we obtain

$$
\begin{gathered}
8 \pi \rho=\frac{1}{\left[1+\gamma-\frac{2 \sqrt{2} \xi_{0} M \cos T}{(1+M \sin T)}\right]} \times \\
\frac{\left[M^{2} \sin ^{2} T+18 M \sin T+14 M^{2}+3\right]}{6(1+M \sin T)^{2}} \\
-\frac{\left[3+10 M^{2}+2 M \sin T-11 M^{2} \sin ^{2} T\right]}{4(1+M \sin T)^{2}} .
\end{gathered}
$$

From Equations (48) and (50), we observe that the positive cosmological constant is a decreasing function of time and approaches a small value in the present epoch.

Some Physical aspects of the Models:

The expansion $(\theta)$ and the $\operatorname{shear}(\sigma)$ in the model (44) are given by

$$
\begin{gathered}
\theta=\frac{2 \sqrt{2} M \cos T}{(1+M \sin T)} \\
\sigma=\frac{1}{\sqrt{3}} \frac{M \cos T}{(1+M \sin T)}
\end{gathered}
$$

When $T \rightarrow 0$ then $\theta \rightarrow 2 \sqrt{2} M$ and when $T \rightarrow \frac{\pi}{2}$ then $\theta \rightarrow 0$. Thus the expansion in the model starts at $T=0$ and it stops at $T=\frac{\pi}{2}$. Since $\lim _{T \rightarrow \infty} \frac{\sigma}{\theta} \neq 0$, hence the models do not approach isotropy for large 
values of $T$. The model has singularity at $T=0$ which is real physical singularity.

\section{Conclusions}

We have obtained a new class of Bianchi type IX anisotropic cosmological models with a viscous fluid as the source of matter. Generally, the models are expanding, shearing and non-rotating. In all these models, we observe that they do not approach isotropy for large values of time $T$.

The cosmological constant in all models given in sections 3.1 and 3.2 are decreasing function of time and they all approach a small positive value as time increases (i.e., the present epoch). The values of cosmological "constant" for these models are found to be small and positive which are supported by the results from recent supernova observations recently obtained by the High - z Supernova Team and Supernova Cosmological Project ( Garnavich et al., 1998 ; Perlmutter et al., 1997, 1998, 1999; Riess et al., 1998; Schmidt et al. , 1998. Thus, with our approach, we obtain a physically relevant decay law for the cosmological constant unlike other investigators where adhoc assumption for the variation were used to arrive at a mathematical expressions for the decaying vacuum energy.

\section{Acknowledgements}

One of the authors (A. Pradhan) thanks to the Inter-University Centre

for Astronomy and Astrophysics, India for providing facility under Associateship Programmes where part of work was carried out. Authors would also like to thank Professor Raj Bali for helpful discussions.

\section{References}

Bali, R. and Dave, S.: 2001, Pramana-J. Phys. 56, 513; 2003, Astrophys. Space Sci. (in press).

Bali, R. and Yadav, M.K.: 2002, pre-print.

Bertolami, O.: 1986, Nuovo Cimento, B 93, 36.

Bertolami, O.: 1986, Fortschr. Phys. 34, 829. 
Burd, A.B., Buric, N. and Ellis, G.F.R.: 1990, Gen. Rel. Grav. 22, 349.

Carroll, S.M., Press, W.H. and Turner, E.L.: 1992, Ann. Rev. Astron. Astrophys. 30, 499.

Chakrabarty, I., Pradhan, A. and Saste, N.N.: 2001, Int. J. Mod. Phys. D 10, 741.

Chakraborty, S. and Nandy, G.C.: 1992, Astrophys. Space Sci. 198, 299.

Dolgov, A.D.: 1983, in The Very Early Universe, eds. Gibbons, G.W., Hawking, S.W. and Siklos, S.T.C. (Cambridge University Press).

Dolgov, A.D., Sazhin, M.V. and Zeldovich, Ya. B.: 1990, Basic Modern Cosmology, (Editions Frontiers).

Dolgov, A.D. and Silk, J.: 1993, Phys. Rev. D 47, 3144.

Dolgov, A.D.: 1993, Phys. Rev. D 48, 2499.

Dolgov, A.D.: 1997, Phys. Rev. D 55, 5881.

Garnavich, P.M. et al.: 1998a, Astrophys. J. 493, L53, Hi-z Supernova Team Collaboration (astro-ph/9710123);

1998b, Astrophys. J. 509, 74, Hi-z Supernova Team Collaboration (astro$\mathrm{ph} / 9806396)$.

Johri, V.B. and Sudarshan, R.: 1988, Phys. Lett. A 132, 316.

Kalyani, D. and Singh, G.P.: 1997, in New Direction in Relativity and Cosmology, eds. V. de Sabbata and T. Singh, Hadronic Press, U S A, p.41.

King, D.H.: 1991, Phys. Rev. D 44, 2356.

Krori, K.D., Chaudhuri, T., Mahanta, C.R. and Mazumdar, A.: 1990, Gen. Rel. Grav. 22, 349.

Maartens, R.: 1995, Class Quantum Gravit. 12, 1455.

MacCallum, M.A.H.: 1979, General Relativity- An Einstein Centenary Survey , Cambridge University Press, London, p. 533.

Murphy, G.L.: 1973, Phys. Rev. D 8, 4231.

Narlikar, J.V.: 1983, Introduction to Cosmology, Jones and Bertlett Publications, Bostan, p. 231.

Narlikar, J.V. and Kembhavi, A.K.: 1980, Fundamental of Cosmic Physics, 6, p. 1.

Padmanabhan, T.: 2003, Phys. Rep. 380, 235; hep-th/0212290.

Padmanabhan, T. and Chitre, S.M.: 1987, Phys. Lett. A 120, 433.

Paternoga, R. and Graham, R.: 1996, Phys. Rev. D 54, 4805.

Pavon, D., Bafaluy, J. and Jou, D.: 1991, Class. Quant. Grav. 8, 357;

1996, "Proc. Hanno Rund Conf. on Relativity and Thermodynamics", Ed. S. D. Maharaj, University of Natal, Durban, p. 21.

Perlmutter, S. et al.: 1997, Astrophys. J. 483, 565, Supernova Cosmology Project Collaboration (astro-ph/9608192);

1998, Nature 391, 51, Supernova Cosmology Project Collaboration (astro$\mathrm{ph} / 9712212)$;

1999, Astrophys. J. 517, 565, Project Collaboration (astro-ph/9608192).

Peebles, P.J.E. and Ratra, B.: 2003, Rev. Mod. Phys. 75, 559; astro-ph/0207347.

Pradhan, A., Sarayakar, R.V. and Beesham, A.: 1997, Astr. Lett. Commun. 35, 283.

Pradhan, A., Yadav, V.K. and Chakrabarty, I.: 2001, Int. J. Mod. Phys. D 10, 339.

Pradhan, A., V.K. Yadav and Saste, N.N.: 2002, Int. J. Mod. Phys. D 11, 857.

Pradhan, A. and Yadav, V.K.: 2002, Int. J. Mod. Phys. D 11, 839.

Pradhan, A. and Aotemshi, I.: 2002, Int. J. Mod. Phys. D 11, 1419.

Pradhan, A. and Pandey, H.R.: 2003,Int. J. Mod. Phys. D 12, 941.

Pradhan, A. and Pandey, O.P.: 2003,Int. J. Mod. Phys. D 12, 1299.

Ratra, B. and Peebles, P.J.E.: 1988, Phys. Rev. D 37, 3406.

Riess, A.G. et al.: 1998, Astron. J. 116, 1009; Hi-z Supernova Team Collaboration (astro-ph/9805201). 
Sahni, V. and Starobinsky, A.: 2000, Int. J. Mod. Phys. D 9, 373; gr-qc/9904398.

Santos, N.O., Dias, R.S. and Banerjee, A.: 1985, J. Math. Phys. 26, 878.

Schmidt, B. P. et al.: 1998, Astrophys. J. 507, 46, Hi-z Supernova Team Collaboration (astro-ph/9805200).

Singh, T., Beesham, A. and Mbokazi, W. S.: 1998, Gen. Rel. Grav. 30, 537.

Tsagas, C.G. and Maartens, R.: 2000, gr-qc/9912022.

Uggla, C. and Zur-Muhlen, H.: 1990, Class. Quant. Grav. 7, 1365.

Vaidya, P. C. and Patel, L.K.: 1986, Praman - J. Phys. 27, 63.

Vishwakarma, R.G. and Abdussattar: 1999, Phys. Rev. D 60, 063507.

Vishwakarma, R.G.: 2000, Class. Quant. Grav. 17, 3833.

Vishwakarma, R.G.: 2001, Class. Quant. Grav. 18, 1159.

Vishwakarma, R.G.: 2001, Gen. Rel. Grav. 33, 1973.

Vishwakarma, R.G.: 2002, Mon. Not. R. Astron. Soc. 331, 776.

Vishwakarma, R.G.: 2002, Class. Quant. Grav. 19, 4747.

Weinberg, S.: 1972, Gravitation and Cosmology, Wiley, New York.

Zeldovich, Ya. B.: 1968, Sov. Phys.-Uspekhi 11, 381.

Zimdahl, W.: 1996, Phys. Rev. D 53, 5483. 